\title{
Reprocessing as a recognition cue
}

\author{
J. EDWARD RUSSO and ROBERT A. WISHER \\ University of California, San Diego, La Jolla, California 92093
}

\begin{abstract}
The use of mental operations as recognition cues was investigated. Two experiments support the hypothesis that processing details are retained in memory and that a re-creation of those processing details can effectively cue recognition. Four subjects performed a mental arithmetic task that emphasized speed and accuracy while discouraging memorization of the numbers. Recognition was cued either by single numbers or by a pair of numbers that, when added, replicated an episode of the original task. Reprocessing an episode was the most effective recognition cue. Of the two single-number cues, the intermediate subtotals were recognized, whereas the numbers that had been physically displayed were not. The study suggests: (1) that the sequence of mental operations is retained in memory, (2) that reprocessing uses this trace to facilitate performance, and (3) that the detection of facilitated reprocessing aids recognition.
\end{abstract}

This research investigates the representation of mental operations in a memory trace. We believe that a memory trace represents a concise record of both symbols and the operations that process and connect these symbols. Furthermore, symbols are better recognized when united with, rather than isolated from, their connecting operations.

First, let us delineate our terms. Borrowing the framework of Newell and Simon (1972), information processing is viewed as the flow of symbols through some central processor. A symbol may have either an external or internal source, depending on whether it is derived from the stimulus or generated by the processor. Directing this flow are operations that select, elaborate, and modify the symbols. The choice of operations depends on the goal of the organism. Cognitive activity is viewed as the complete sequence of operators and symbols used during task performance.

There is little doubt that some parts of the cognitive activity can be remembered, e.g., very recent or salient symbols. The critical claim is that all elements of this activity are preserved in an ordered sequence that reflects the original processing. This view of memory is at least as old as Koffka's (1935) "trace column." It is currently advocated by Craik and Tulving (1975), Jacoby (1974), Kolers and Ostry (1974), Murdock (1974), Neisser (1967), and Posner and Warren (1972), among others. Beyond the issue of whether cognitive acts are recorded in memory is whether these operations

The order of authors is alphabetical; their contributions were equal. The authors express their appreciation to Barbara Greenlee for experimental assistance and to Barbara Dosher and James McClelland for comments on an earlier draft. This research was partially supported by NIH Grant MH-24880 and by NIH Grant MH-15828 to the Center for Human Information Processing, University of California, San Diego. Requests for reprints should be sent to J. Edward Russo, Graduate School of Industrial Administration, Carnegie-Mellon University, Pittsburgh, Pennsylvania 15213. can aid recognition. This latter issue, operations as recognition cues, is the focus of the present paper.

We claim that access to a memory trace is facilitated when its corresponding cognitive activity is re-created. A re-creation occurs whenever both the symbols and the operations linking those symbols are reproduced. Alone, the symbols might be insufficient for recognition. However, when embedded in their original processing environment, a richer stimulus pattern emerges. Recognition is facilitated to the extent that this extra-symbolic information is used.

Bartlett's (1932) concept of memory schemata considered the value of cognitive acts as memory cues. In the study most relevant to the present research, Kolers (1973) presented sentences in either conventional or altered formats. He found that recognition was highest when sentences were presented in the same format. This effect of format occurred even though his subjects had been instructed to recognize only meaning. Kolers concluded that a subject remembers the perceptual operations performed during comprehension. Although Kolers' study varied processing independently of the task goal, the sequence of operations was not controlled. Thus, one cannot know whether the original processing was duplicated.

The central hypothesis of the present study is that a match between the cognitive activity during the initial task and during recognition can serve as an effective recognition cue. Such a match is obtained by requiring a reprocessing of the original stimulus, thereby reconstructing a portion of the initial processing sequence. Two predictions follow from this claim: First, reconstructing the original sequence of symbols and operations should cue recognition more effectively than the same symbols presented in isolation; second, reproces. sing should cue recognition even when no discriminatory information is available from the individual signals. These two predictions are tested in Experiment I. 
Table 1

Proportion of Correct Recognitions for Single Numbers

\begin{tabular}{ccc}
\hline Subject & External & Internal \\
\hline 1 & $.46 \dagger$ & $.72^{*}$ \\
2 & .53 & .56 \\
3 & .52 & $.64^{*}$ \\
4 & .54 & $.70^{*}$ \\
Mean & .51 & $.66^{*}$ \\
\hline
\end{tabular}

$* p<.05$.

†Each proportion was based on approximately 95 observations (range: 85 to 112). The only exception was the proportion for external numbers for Subject 2 (.53). Because of a change in strategy during Session 11, this proportion was based on the 50 observations made during Sessions 1 and 2. See Note 1 in the text for details.

\section{EXPERIMENT I}

\section{Method}

Essential to the experimental strategy was a task whose sequence of operations was well known and would show little change over two identical trials. Simple arithmetic met these criteria. Eight two-digit numbers were alternately added and subtracted from an initial value, with only the final total reported. The nine numbers that were displayed visually constituted the external symbols. Generated during the arithmetic episodes were seven subtotals, or internal symbols, and the final total. A sample trial, with the generated subtotals shown in parentheses, was: $74,-25,(49),+27,(76),-18,(58),+24,(82),-19$, (63), $+16,(79),-22,(57),+15,72$.

Each trial was followed by one of two recognition tests. In the first type of test the subject was shown a subtotal followed by two addends, from which two alternative episodes could be constructed. From the test display (76) -23 , the subject could generate two episodes, $76-23(=53)$ and $76-18(=58)$, only one of which occurred during the preceding trial. The distractor episode had both an incorrect external addend $(-23)$ and an incorrect internal subtotal (53). Either distractor number could have been incorrect in two ways: not present in the original trial (the NP condition) or present but located elsewhere (the $P$ condition). For example, the distractor $76-19(=57)$ had both components present in the trial but not immediately following the 76. Varying the NP and P conditions independently for the external addends (Ex) and internal subtotals (In) led to four distractor conditions $\left(\operatorname{Ex}_{\mathbf{N P}}-\mathrm{In}_{\mathrm{NP}}, \operatorname{Ex}_{\mathrm{P}}-\mathrm{In}_{\mathrm{NP}}, \operatorname{Ex}_{\mathbf{N P}}-\mathrm{In}_{\mathbf{P}}\right.$, and Exp-InP).

In the second type of recognition test, the subject was shown a pair of numbers, either two external numbers or two internal subtotals. Only one of the numbers had appeared during the trial just completed. For the sample trial shown above, a test might have been -23 for external or 79 for internal numbers. These recognition accuracies provided base rates for the various episode conditions.

Stimuli. The external numbers lay between 12 and 28 , excluding 20 . The eight numbers in any trial were equally divided between odd and even and between above and below 20 , and none was repeated. The appropriate sign, + or -, appeared to the left of each number. The signed numbers were displayed successively in the center of a CRT (Tektronix 611). The starting number was always greater than 60 . The internal numbers were bounded by 31 and 99 , with none divisible by 10 and none repeated. In the recognition tests, all distractors fell within the range of numbers that had occurred in the preceding trial.

There were two stimulus sets containing recognition tests of single numbers and four sets testing recognition for episodes. Each set contained 60 trials, occupied one session, and was repeated once. The 4 sessions testing recognition of the single numbers were the first 2 and last 2 of the 12 postpractice sessions.

Subjects. Four naive student volunteers served as subjects. Subjects 1 and 3 were female; Subjects 2 and 4 were male. Each subject participated in 14 sessions of 1 -h duration. Payment was based upon performance and averaged $\$ 2.25$ per session.

Procedure. A payoff scheme was designed to emphasize speed in completing the arithmetic task and to make the recognition task seem less important. A correct answer on the arithmetic task earned 3 cents, while an error cost 3 cents and disqualified the subject from the remaining payoff opportunities. The time to complete each arithmetic sequence was compared to each subject's mean performance on previous sessions and determined a reward between +2 and -2 cents. Because of the decrease in processing time with practice, the time criterion for the speed payoff was adjusted between sessions. Finally, the recognition response was worth +1 or -1 cents for correct or incorrect answers.

In order to determine an initial time criterion, the first session contained 40 trials with no recognition tests. A second session provided practice on the three types of recognition tests. Each of the 12 remaining sessions began with five warm-up trials.

Before each trial, the word READY appeared on the screen. The subject started a trial with a buttonpress, and the initial value was displayed. The subject depressed a button to display the next number, performed the arithmetic, pressed the button for the next number, etc. When the task was completed, the screen was erased and the subject entered the answer via a keypunch. If the final total was correct, a recognition test followed. If an incorrect answer was entered, the word NO appeared with the correct answer below it, and no recognition test was given. On correct trials the time between task completion (the last buttonpress) and the presentation of the recognition test stimulus was $6 \mathrm{sec}$. The experimental procedure was controlled by a PDP-1 2 laboratory computer (Digital Equipment Corporation).

Separate buttons designated the top and bottom alternatives in the recognition test. There was no limit on time to respond. After the recognition response, the subject was told the payoff for speed and recognition on the current trial and the cumulative payoff for the session. When the subject was satisfied with this information, he pressed a button to start the next trial.

Under the preceding procedure and payoff scheme, the average time to complete the eight arithmetic operations per trial was $17.0 \mathrm{sec}$ (range: 13.7, 20.7). The mean rate of errors in the reported total was .13 (range: $.06, .17$ ).

\section{Results}

The proportions of correct recognitions for the two single-number cues are shown in Table 1. For external numbers the mean accuracy over subjects was .51 , a value not significantly different from chance. (The .05 level of significance was used throughout). This failure to recognize external numbers held for all four subjects individually. ${ }^{1}$

When a single internal number was used as a cue, the mean proportion of correct recognitions was .66 , significantly above chance $(z=6.58)$. This effect held individually for three of the four subjects (range of $z: 2.84$ to 4.78), with only Subject 2 failing to recognize the generated subtotals. Thus Subjects 1,3 , and 4 seemed to remember internal subtotals, while not remembering the external stimulus at all. Subject 2 did not remember any single numbers at a level reliably above chance. 
Episodes as cues. If a memory trace is a blend of symbols and operations, then together they should serve as a more effective recognition cue than the symbols alone. In this case, episodes should be better recognized than the numbers alone. This hypothesis can be tested in two ways. First, consider the condition where both numbers in the distractor were present (the Exp-Inp condition). Here, remembering only single numbers could not have aided recognition. Unless the episodic relation between numbers was recognized, chance performance would have been expected. However, the recognition rates for this condition (see Table 2) were significantly above chance for all subjects (range of $\mathrm{z}$ : 2.00 to 6.86 ).

A second test compares the ExNP-InP against the external base rate and the Exp-InNP against the internal base rate. Thus, for Subject 1, .66 was compared to 46 , and .88 to .72 . If discrimination were based on singlenumber cues, then no differences were expected. Of these eight tests for the difference between two proportions, seven showed episodic cuing to be significantly superior to the appropriate single cue (range of z: 2.76 to 6.16). The results of both sets of tests supported the hypothesis that reprocessing serves as a more effective cue. Note, however, that these data might also have been accounted for by remembering only the adjacency of numbers in the sequence and not by reprocessing. This explanation is considered shortly.

Single numbers within episodes. The episodic cuing data provided an additional demonstration of the failure of external numbers as cues. The effect of the external numbers could be isolated by varying only the presence of the external distractor, i.e., by comparing the Exp-InP and ExNP-InP conditions and by comparing the Exp-InNP and ExNP-InP conditions. All eight tests for a difference between two proportions showed that external numbers had no significant effect on recognition. The mean accuracies for external numbers not present and present were .79 and .80 , respectively.

In a similar manner, the effectiveness of internal numbers as cues was tested by comparing the Exp-InP and Exp-InNP conditions and by comparing the $\operatorname{Ex}_{N P-I n P}$ and $\operatorname{Ex}_{N P}-\operatorname{In}_{N P}$ conditions. When the

Table 2

Proportion of Correct Recognitions for Episodes

\begin{tabular}{ccccc}
\hline Subject & \multicolumn{4}{c}{ Distractor Condition } \\
& $\operatorname{Ex}_{\mathbf{p}}-\mathrm{In}_{\mathbf{p}}$ & $\mathrm{Ex}_{\mathrm{NP}}-\mathrm{In} \mathbf{p}$ & $\mathrm{Ex}_{\mathbf{p}}-\mathrm{In}_{\mathrm{NP}}$ & $\mathrm{Ex}_{\mathrm{NP}}-\mathrm{In}_{\mathrm{NP}}$ \\
\hline 1 & $.63^{* \dagger}$ & .66 & .88 & .84 \\
2 & $.60^{*}$ & .61 & .75 & .75 \\
3 & $.85^{*}$ & .92 & .96 & .92 \\
4 & $.76^{*}$ & .75 & .93 & .86 \\
Mean & $.71^{*}$ & .74 & .88 & .84 \\
\hline
\end{tabular}

$* p<.05$.

tEach proportion was based on approximately 96 observations (range: 89 to 112).
Table 3

Recognition as a Function of Temporal Spacing Between the Correct and Distractor Subtotals

\begin{tabular}{cclllll}
\hline Spacing & \multicolumn{2}{c}{ All } & \multicolumn{2}{c}{ Positive } & \multicolumn{2}{c}{ Negative } \\
\hline 1 & .74 & $(114)^{*}$ & .80 & $(86)$ & .54 & $(28)$ \\
2 & .78 & $(224)$ & .80 & $(107)$ & .75 & $(117)$ \\
3 & .75 & $(259)$ & .74 & $(106)$ & .75 & $(153)$ \\
4 & .56 & $(87)$ & .56 & $(87)$ & & $(0)$ \\
\hline
\end{tabular}

*The number of observations on which the adjacent proportion was based appears in parentheses.

internal distractor was not present, the episodic cue was significantly more effective on seven of eight tests (range of $\mathrm{z}: 1.92$ to 4.03 ). Over subjects, the mean recognition rates were .86 and .72 for the not-present and present conditions, respectively. Both of these results agreed with the earlier findings for single numbers as cues-orly the internal subtotals were recognized.

The use of temporal cues. Although the above results supported the reprocessing hypothesis, they did not preclude an explanation based only on temporal cues. Suppose that each internal subtotal was tagged as occurring early, middle, or late in the sequence. During the recognition test, the temporal tags of the two generated subtotals could have been compared to the tag of the common subtotal. For instance, if a subject remembered that the common subtotal appeared early in the sequence, he could have eliminated a generated subtotal tagged middle or late. Note that this explanation depended on memory for individual symbols and their relative positions, unlike the reprocessing hypothesis which required memory for an amalgam of symbols and operators.

The temporal tag hypothesis was tested by examining the conditions in which the internal distractor was present (Exp-Inp and $\left.\operatorname{Ex}_{N} \mathrm{P}-\mathrm{In}_{\mathrm{P}}\right)$. If recognition was based only on the proximity of subtotals, then greater temporal spacing between the correct and distractor subtotals should have produced more accurate discrimination. Temporal spacing was defined as the number of subtotals between the distractor subtotal and the correct episode. The recognition rate for each of the four levels of spacing is presented in the column labeled "All" in Table 3. These proportions infirmed the hypothesis that larger temporal spacing would lead to better recognition. Not only did they not increase monotonically with spacing, but the significant drop $\left[\chi^{2}(3)=16.3\right]$ at a spacing of 4 was opposite to the prediction.

A different use of temporal cues was suggested by the subjects' strategies. Upon completing the experiment, each subject was asked for his recognition strategy. All four subjects reported basing their recognition entirely on the internal subtotals. No one mentioned either external numbers or the reprocessing of episodes. Given the reported strategy, recognition might have been based on the relative recency of the two subtotals. Such a strategy is closely related to backward scanning, which 
Table 4

Recognition Accuracy as a Function of Serial Position

\begin{tabular}{lccccc}
\hline & \multicolumn{5}{c}{ Serial Position } \\
& 2 & 3 & 4 & 5 & 6 \\
& Add & (Subtract) & Add & (Subtract) & Add \\
\hline $\begin{array}{l}\text { External Number } \\
\text { Internal Number }\end{array}$ & $.47^{*}$ & .52 & .48 & .57 & .56 \\
$\begin{array}{l}\text { Episode } \\
\ln _{\text {NP }}\end{array}$ & .60 & .59 & .68 & .76 & .70 \\
In & .79 & .90 & .84 & .85 & .90 \\
& .70 & .74 & .72 & .72 & .72 \\
\hline
\end{tabular}

* The number of observations on which the proportions were based ranged between 69 and 76 for external numbers, between 78 and 86 for internal numbers, and between 159 and 176 for each of the 10 episode proportions.

Jacoby (1974) proposes as the retrieval process for immediate episodic memory. Note that our experimental design may have encouraged a recency comparison strategy. For the trials when the distractor had not been present (the two InNP conditions), a correct recency judgment would have always produced correct recognition. For all $\ln _{\mathrm{p}}$ trials, however, relative recency was independent of recognition accuracy. (The data in Table 3 come only from these latter trials.)

To test the recency hypothesis, the data in Table 3 were partitioned into two groups. A positive temporal spacing indicated that the distractor followed the episode, while a negative spacing meant the opposite. For positive spacing, a recency strategy predicted worse recognition as temporal spacing increased. For negative spacing, the prediction was reversed. The data in Table 3 confirmed both predictions. The predicted differences were statistically significant for positive spacing $\left[\chi^{2}(3)=17.5\right]$, but only marginally so for negative spacing $\left[\chi^{2}(3)=5.8, p=.054\right]$.

These results implied that subjects were able to use recency information. They did not appear to use more sophisticated temporal cues. Note, however, that recognition was not based entirely on a recency comparison. If that had been true, then recognition when the internal distractor was not present $(M=.86)$ should not have differed from the base rate $(M=.66)$. Obviously, subjects did more than compare recency.

Factors affecting recognition. Several factors might have intluenced the cuing effectiveness of individual episodes. Recognition accuracy as a function of serial position is shown in Table 4. Because only the internal numbers were effective cues, the episodes were combined over the Ex $x_{N P}$ and Exp conditions. A small serial position effect was observed for each of the three types of cues. This effect was confined mainly to a decline at the earlier positions: Positions 24 for external numbers, Positions 2-3 for internal numbers, and Position 2 for episodes. Using a test for the difference between two proportions, mean recognition over the earlier serial positions was significantly lower only for $\operatorname{In}_{\mathrm{NP}}$ episodes $(z=3.73)$ and for internal numbers $(z=2.43)$. Note that the use of a recency comparison suggested that there should have been a serial position effect for these two cues. Only for these cues was the correct internal subtotal compared with a distractor that had not been present. Since the recency of the correct subtotal increased with serial position, recognition should have been better at the more recent serial positions.

A longer duration of the original processing of an episode could have established a stronger trace. Thus, the original processing time might have been positively related to recognition accuracy. To test this, each cuing episode was classified as leading to a correct or incorrect recognition. The original processing times of these episodes were averaged within the correct and incorrect categories. The mean processing durations were $2.07 \mathrm{sec}$ and $2.03 \mathrm{sec}$ for correct and incorrect, respectively. These durations were not reliably different $(t=.31$, $\mathrm{df}=1,644$, pooled est $\sigma=1.44$ ). This same comparison was made for individual subjects, separately for addition and subtraction episodes. None of the eight pairs of mean durations showed a significant difference. This finding confirms other failures to predict recognition from processing duration, or time on task (Craik \& Tulving, 1975; Kolers, 1973; Kolers \& Ostry, 1974).

The results of Experiment I supported the claims that processing details are retained in memory and that reprocessing can effectively aid recognition. On the other hand, there was clear evidence that recognition was also based on a recency comparison between the two internal subtotals. In addition, all subjects reported basing their strategies on internal numbers only and not on the reconstruction of episodes. What would happen if internal numbers were removed as recognition cues? If subjects were prevented from using such cues, then a more episodic or reconstructive strategy might emerge. This rationale formed the basis for Experiment II.

\section{EXPERIMENT II}

\section{Method}

This experiment differed from Experiment $I$ in two ways. First, the format of the recognition test was altered slightly to two sequences that both added (or subtracted) to the same number, e.g., $63+16$. Second, the initial number in each sequence was always a member of the set of generated subtotals. That is, subjects were shown only episodic distractors whose internal number had appeared in the original sequence (the

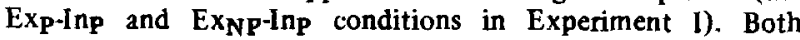
changes were designed to reduce the role of generated subtotals as recognition cues.

The four subjects used in Experiment $I$ also participated in Experiment II. Two new sets of 60 trials each were constructed. Each set required one session. The pay off and other procedures were unchanged from Experiment I.

\section{Results}

The recognition accuracies (see Table 5) again supported the hypothesis that reprocessing an episode facilitated recognition. All subjects exhibited abovechance performance in both the ExNP and Exp condi- 
tions (range of $z: 1.69$ to 5.89 ), as well as no significant difference between these two conditions. When these proportions were averaged and compared with the corresponding values from Experiment I, they showed a small but significant decrease in recognition accuracy, from .72 to $.66(\mathrm{z}=1.67)$. This decrease was significant for Subjects 3 and 4 only ( $z=2.03$ and 2.53 , respectively).

A possible explanation for this decline involved the change in the format of the recognition test. The new format allowed the missing subtotal to be computed from one episode only. Thus, even though subjects were instructed to add (subtract) both sets of numbers, only one computation was needed. Consequently, there was no guarantee that the target episode was always reprocessed. A decrease in the frequency of reprocessing could have explained the observed drop in recognition accuracy.

There should have been no recency effect in Experiment II, because subjects knew that all internal distractors had occurred in the original sequence. The relevant data are presented in Table 6 . Recall that a recency comparison led to better recognition as (absolute) temporal spacing increased. The accuracies in Table 6 provided only weak support for this hypothesis. The general trend conformed to the recency prediction, but marked deviations from the predicted monotone relation occurred. These results seemed to imply that the recency strategy observed in Experiment I was inhibited but not completely eliminated.

Eliminating the presence of the internal subtotal as a cue should have altered subjects' strategies. Indeed, Subjects 1, 2, and 4 reported having more difficulty with the new task and were unable to devise a satisfactory strategy. These subjects occasionally reported the recognition of an addition or subtraction within an episode, but they were no more certain of these than of other recognitions. Subject 3 reported coupling each subtotal to the common subtotal and basing recognition on the ordered pairs. The important finding here is that, despite their ability to recognize, Subjects 1,2 , and 4 could not identify the information on which their recognitions were based. If reprocessing was providing

Table 5

Recognition Accuracy Without Internal Numbers as Cues

\begin{tabular}{cccccc}
\hline & \multicolumn{3}{c}{ Experiment II } & \\
\cline { 2 - 5 } Subject & Ex $_{\text {NP }}$ & Ex $_{\mathbf{p}}$ & $\begin{array}{c}\text { Com- } \\
\text { bined }\end{array}$ & $\begin{array}{c}\text { Experi- } \\
\text { ment I }\end{array}$ & $\begin{array}{c}\text { Dif- } \\
\text { ference }\end{array}$ \\
\hline 1 & $.60^{* \dagger}$ & $.60^{*}$ & .60 & .64 & -.04 \\
2 & $.66^{*}$ & $.64^{*}$ & .65 & .60 & .05 \\
3 & $.82^{*}$ & $.76^{*}$ & .79 & .88 & $-.09^{*}$ \\
4 & $.58^{*}$ & $.66^{*}$ & .62 & .76 & $-.14^{*}$ \\
Mean & .66 & .66 & .66 & .72 & $-.06^{*}$ \\
\hline
\end{tabular}

${ }^{*} p<.05$.

tThe proportions in the first two columns were based on between 44 and 54 observations.
Table 6

Recognition as a Function of Temporal Spacing Between Correct and Distractor Subtotals

\begin{tabular}{ccccc}
\hline Spacing & \multicolumn{2}{c}{ Positive } & \multicolumn{2}{c}{ Negative } \\
\hline 1 & & $(0)^{*}$ & .64 & $(25)$ \\
2 & .73 & $(52)$ & .60 & $(75)$ \\
3 & .53 & $(92)$ & .75 & $(73)$ \\
4 & .81 & $(27)$ & & $(0)$ \\
\hline
\end{tabular}

*The number of observations on which the adjacent proportion was based is shown in parentheses.

the critical information, subjects were uniformly unaware of it.

\section{Discussion}

The present research differs from typical memory experiments in both its initial task and memory prote. In traditional procedures the main task is to memorize a set of items. Repeated presentations or rehearsals increase the saliency of to-be-remembered items, while obscuring the sequence of operations associated with any single processing of an item. In the present experiments, the subjects' main task was to perform arithmetic computations as rapidly as possible. This makes deliberate memorization of the individual numbers both difficult and unprofitable. A second difference occurred in the type of recognition probe. Usually only single items are presented for recognition. Here, however, subjects reprocessed a complete episode of the original arithmetic task. We believe that both reducing the saliency of single items and using reprocessing as a recognition cue were essential to the present findings.

The experimental results may be summarized as follows. First, processing detail beyond individual symbols is retained in memory. Second, the act of reprocessing permits the stored details to be used for recognition. An important implication of this result is that reprocessing must have used sequential information, i.e., must have taken advantage of temporally based associations between the individual numbers. Third, subjects were uniformly unaware of the value of reprocessing. In neither experiment did subjects report strategies that recognized the usefulness of reprocessing. Finally, the internal subtotals were remembered, whereas the externally displayed numbers were not. The remainder of this discussion proposes a theoretical explanation for the above phenomena.

Reprocessing as facilitated performance. The present experiments suggest that the memory trace records all cognitive activity, including both the individual symbols and the set of connections between them. This view of trace formation resembles closely both those of Craik and Jacoby (1975) and Murdock (1974). The critical question, however, is not the nature of this trace but how recognition can be based on this stored record of processing activity.

We claim that accessing the trace facilitates a second 
execution of the same sequence of operations. We claim, further, that recognition is based on the detection of this facilitated reprocessing. That is, the subject can sense that the second processing is easier and faster. Empirical support for the first of these claims, facilitated reprocessing, is provided by Rosen (Note 1). When his subjects re-added four digits, mean task time dropped from $2.02 \mathrm{sec}$ to $1.67 \mathrm{sec}$. This same study sheds some light on our second claim: that facilitated reprocessing can be detected and attributed to memory. Rosen recorded eye fixations on each of the four digits to be added. The durations of these fixations indicated an accelerating pattern of facilitation. That is, the durations became increasingly brief (relative to initial processing time) as the task progressed. Rosen (Note 1) interpreted this and related results as indicating an increasing reliance on memory (internal) control of the reprocessing and a reciprocal decrease in perceptual (external) control. The relevance of this result for the present studies is that facilitated reprocessing is intimately linked with reliance on the memory trace. It seems reasonable, therefore, that the facilitation induced by reprocessing can serve as a basis for recognition. It is also worth noting that Rosen's subjects could not report important aspects of their reprocessing, much as our subjects were unaware that their recognitions were based on reprocessing.

Processing contiguity. The connections between numbers in the memory trace are based on the detailed sequence of operations that enables each two-digit addition. For example, the addition of 63 and 16 probably requires a sequence of operations like: $6+3=9$, hold 9 , carry $=0,1+6=7$, recall 9 , subtotal $=79$. The actual summing of numbers, like 6 and 3 , may be further represented as the sequence: select 6 , increment by 1 , increment by 1 , increment by 1 , total $=9$ (Groen \& Parkman, 1972). The above operations may, in turn, be constructed from a set of (unobservable) elementary information processes (Newell \& Simon, 1972). The essential point here is that the bonds between numbers like 63,16 , and 79 are not formed by temporal contiguity alone but are themselves constructed from an activated sequence of operations.

The distinction between reprocessing and temporal contiguity as explanations of the preceding results can be illustrated by the following thought experiment. Suppose that the recognition format had been $63+1679$. Suppose also that the subject could be prevented some. how from re-adding the numbers. A temporal contiguity hypotheses predicts that the juxtaposition of 63 , +16 , and 79 would be just as effective as recomputing $63+16=79$. To the contrary, we believe it is apparent that the juxtaposed numbers would not provide as complete a cue as would reprocessing. Unfortunately, a subject cannot be prevented from re-adding 63 and 16 in the above stimulus, so this experiment could not be performed.

Memory for external vs. internal symbols. The present experiments show good memory for internal numbers, with little or no memory for the physically presented numbers. The two groups of numbers were very similar, so it is unlikely that one set was naturally easier to recognize. However, the processing of the two types of symbols differed in important ways. The generated subtotals needed to be retained, at least long enough for the subject to press a button to call the next number to be added. The external numbers, on the other hand, were perceived, processed, and discarded. This distinction may have accounted for the recognition of internal but not of external symbols. The longer active processing of the internal numbers could have permitted the spread and elaboration processes that Craik and Tulving (1975) argue are advantageous to retention.

Reprocessing as a memory probe. Tasks that probe remembered information vary widely in difficulty. Recall is generally more difficult than recognition, and reconstruction is an especially difficult version of recall. The reprocessing task used in the present experiments lies on the other end of the continuum of difficulty; it can be regarded as a special version of recognition. Reprocessing forces a re-creation of the original cognitive activity, providing the subject with more information than is available in typical recognition tasks.

Just as reprocessing extends the continuum of memory probes, it also lies outside current categorizations of "retrieval processes." For example, Craik and Jacoby (1975; see also Jacoby, 1974) argue for two such processes. To retrieve from semantic memory, the probe information is combined with stored rules. To retrieve from episodic memory, as in the present experiments, a backward search is conducted until the target item is detected. In contrast to both of these procedures, reprocessing may not require any retrieval (in the strict sense). The to-be-remembered episode is completely re-created, and the subject need only match this information with the memory trace. Thus, the "retrieval process" engendered by reprocessing should be added to current lists of such processes.

The preceding discussion points out the unique role of reprocessing as a memory probe. It provides the subject with more information than other memory probes, and it seems to rely on a different type of retrieval process. Both of these features make the reprocessing technique uniquely valuable for studying the most common and fragile of all memories-immediate, complete, episodic memory.

\section{REFERENCE NOTE}

1. Rosen, L. R. Memory traces of transient cognitive processes. Unpublished doctoral dissertation, University of California, San Diego, 1975.

\section{REFERENCES}

Bartlett, F. C. Remembering. Cambridge: Cambridge University Press, 1932. 
CRAIK, F. I. M.. \& JACOBY, L. L. A process view of short-term retention. In F. Restle, R. M. Shiffrin, N. J. Castellan, H. R. Lindman \& D. B. Pisoni (Eds.), Cognitive theory (Vol. 1). Hillsdale, N.J: Lawrence Erlbaum, 1975.

Craik, F. I. M., \& Tulving, E. Depth of processing and the retention of words in episodic memory. Journal of Experimental Psychology: General, 1975, 1, 268-294.

Groen, G. J., \& Parkman, J. M. A chronometric analysis of simple addition. Psychological Review, 1972, 79, 329-343.

JACOBY, L. L. The role of mental continguity in memory: Registration and retrieval effects. Journal of Verbal Learning and Verbal Behavior, 1974, 13, 483-4\%.

KoffKa, K. Principles of gestalt psychology. New York: Harcourt \& Brace. 1935.

Kolers, P. A. Remembering operations. Memory \& Cognition, 1973, 1. 347-355.

Kolers, P. A., \& Ostry, D. J. Time course of loss of information regarding pattern analyzing operations. Journal of Verbal Learning and Verbal Behavior, 1974, 13, 599-612.

MURDock, B. B., JR. Human memory: Theory and data. Hillsdale, N.J: Lawrence Erlbaum, 1974.

Neisser, U. Cognitive psychology. New York: AppletonCentury-Crofts, 1967.

Newell, A., \& Simon, H. A. Human problem solving. Englewood Cliffs, N.J: Prentice-Hall, 1972.

PosNer, M. I., \& Warren, R. Traces, concepts, and conscious constructions. In A. Melton \& E. Martin, (Eds.), Coding process in human memory. Washington, D.C: Winston, 1972.

\section{NOTE}

1. As noted in Table 1 , the tests of external numbers during the final two sessions were dropped from Subject 2's data. When we tested for differences in accuracy between the first two and last two sessions, only the external numbers for Subject 2 exhibited a significant difference at the liberal .10 level. From a mean of .53 over the first two sessions, his accuracy rose to .61 during Session 11 and to .74 during Session 12 . When asked about his strategies for single numbers, Subject 2 admitted discovering a useful mnemonic during Session 11. Because he was an avid sports fan, he claimed to associate certain numbers with the names of ballplayers. For example, he said whenever 24 was presented in the recognition test, he tried to remember whether Willie Mays had appeared in the original sequence. Because almost all of his eight special numbers were less than 30 , this mnemonic worked only for external numbers. The episodic data were not affected because this strategy was adopted after those data had been collected.

(Received for publication January 13.1976; revision accepted April 1. 1976.) 